\title{
Model organisms on roads less traveled
}

Beyond the well-known pantheon of model organisms are others. A shift is underway to level the playing field.

\author{
Vivien Marx
}

I I hat model do you work on?" is a question that can start a spirited conversation at a conference networking event. The query might lead to feigned interest and the chat fizzles. But social situations of this kind, once they return after the pandemic, need not unfold in such a polarized way. In some ways, history is coming full circle: studying diversity and complexity is modern. Many labs do so by working with organisms not in the pantheon of classic models.

Historically, as University California, Irvine biologist Rowland Davis noted ${ }^{1}$, in the eighteenth and nineteenth centuries, amid a growing appreciation for evolutionary mechanisms, biologists used observational studies to tease out patterns that underpinned diversity, complexity and organismal development. The twentieth century's advent of genetics led to experimentalists focusing on inheritance, and they used a few model organisms to do so. Corn was set up as a model in 1900, mouse and fruit fly a few years later; others followed. Research on species outside the pantheon of models continued but faded from view. Over time, notes Davis, methods were developed that are used to expand on the insight from the few 'genetically domesticated' model organisms. "The advent of genomics frees geneticists from their confinement to single species." Now, functional and comparative analysis of genes and entire genomes are taking twenty-first-century biologists back to probing diversity and complexity in organisms.

Before the genomes of many organisms were known, when genome editing was not yet possible, these classic models were the only ones amenable to genetics experiments. These days, considering only the classic model organisms is "a very old-fashioned way of thinking," says Paola Oliveri, a researcher at University College London. Perhaps, she says, the land-living organisms of the pantheon were chosen because humans, too, live on land, and we feel more connected to them. But many species on Earth inhabit marine ecosystems. She thinks a greater appreciation of marine model organisms will emerge as "we hopefully will understand that human health means also ecosystem health, world health." As sequencing dropped in price, its use spread. Along with other

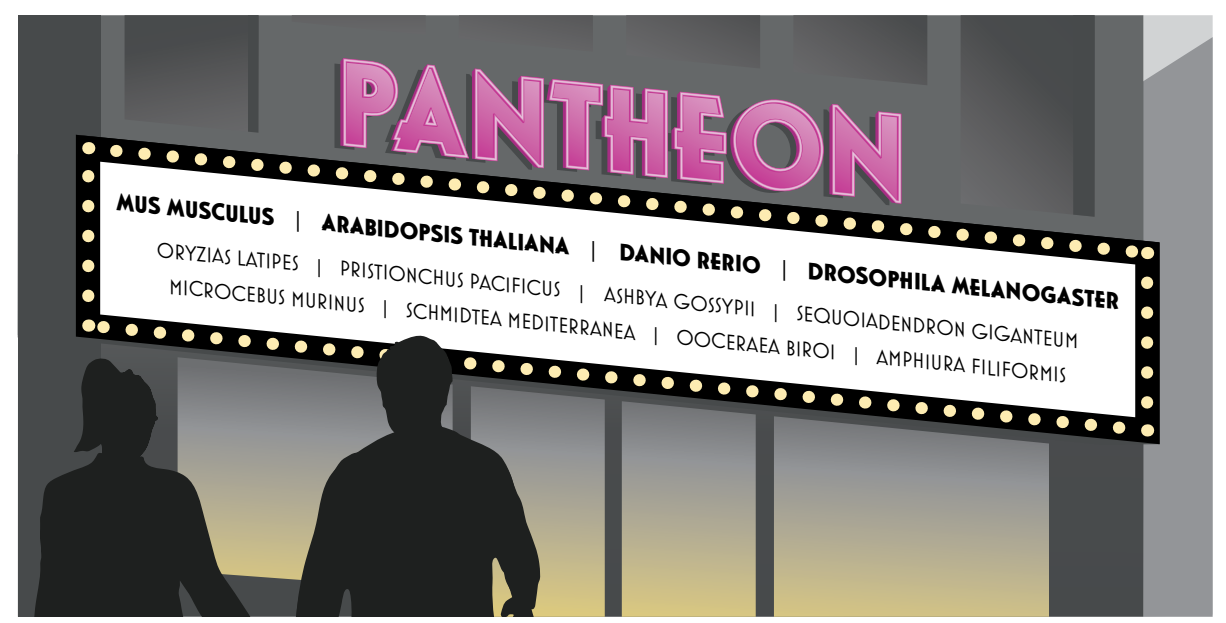

For the pantheon of model organisms, there's a wealth of data. Some labs choose to work with less well-known research organisms even though fewer resources are available. Credit: Erin Dewalt, Thomas Phillips, Springer Nature

tools, researchers could explore many types of questions in multiple species, widen their gaze and avoid being tempted to think what happens in one experimental organism is "the truth," says Oliveri. For example, in the fruit fly embryo, the protein bicoid patterns the animal's axis through a concentration gradient from anterior to posterior, but it turns out not to form a gradient pattern in all insects.

A common strategy has been to focus on a handful of species that yield compliantly to laboratory domestication, but it's unlikely that these species suffice to uncover all the principles that underpin life, notes Howard Hughes Medical Institute investigator Alejandro Sánchez Alvarado 2 , who is the executive director and chief scientific officer of the Stowers Institute for Medical Research.

The focus on a few organisms has led fields in biology to become highly specialized endeavors that promise mechanistic understanding of some of the most difficult questions in biomedical research today, he says. Yet this specialization may have begun to impede progress in science "at best, and at worst, led us astray." It's time to abolish this split between models and non-models and use the term 'research organism', he says, to now take the "technological armamentarium" that includes genome editing, imaging, bioinformatics, high-throughput assays and automation to explore life's wealth and interrogate organisms and entire ecosystems previously inaccessible to effective inquiry.

Labs can pursue a question with a "mature research organism," as he calls an established model. "But what if that biology is under-represented in the available systems?" That's when one must look to the natural world and see which organism might be exaggerating the attribute a researcher seeks to study. A scientist can find an experimental vulnerability and exploit it to get an answer to a problem of interest. "In fact, how do you think the five to seven research organisms most broadly used today were selected?" says Sánchez Alvarado. “They were all exaggerators of the problems the scientists of the time wanted to study."

\section{Planaria, Schmidtea mediterranea} It intrigues Sánchez Alvarado that some animals, but not others, can regenerate missing or damaged tissue or body parts, such as their brain, their head, their digestive system. He and colleagues have found that in the body plan of planaria, or flatworms, are undifferentiated adult stem cells that enable this ability.

The key genetic tool he and his group have been using to manipulate planarians since 1998 is RNAi, or RNA-mediated genetic interference. They have also adopted ways 


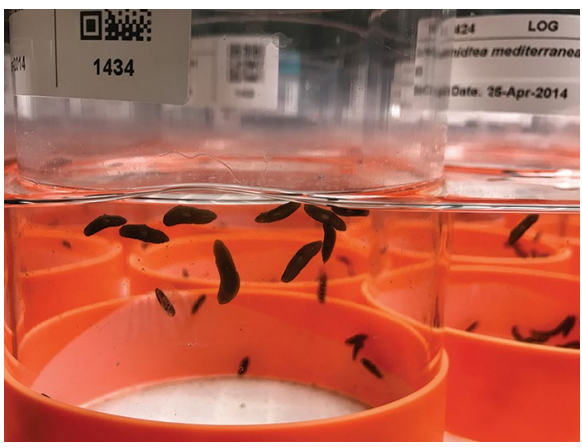

Sánchez Alvarado lab/HHMI, Stowers Institute for Medical Research.

to see gene expression or cell behavior and approaches for quantifying gene expression in toto and all the way down to single cells, he says. He and others want to expand this repertoire to permanent genome modifications, he says. "The prospective isolation of planarian stem cells and the advent of CRISPR-Cas has re-energized all of us to continue our efforts to develop such methods to study the important and remarkable biology of planarians." In his view, "regeneration is the last wild frontier of developmental biology" and it has the potential to solve complex biology problems in the $21^{\text {st }}$ century. Beyond obvious potential practical ramifications related to human health, he says, regeneration provides fertile, largely unexplored vistas to obtain a greater understanding of the fundamental genetic, molecular, and cellular activities that govern biological function.

\section{Brittlestar Amphiura filiformis}

"We all have our special species," says Oliveri. Hers are amid echinoderms - the sea urchin and the brittlestar. She uses them for comparative genomics, among other things. The sea urchin has lent itself beautifully to many discoveries, she says, and its genome

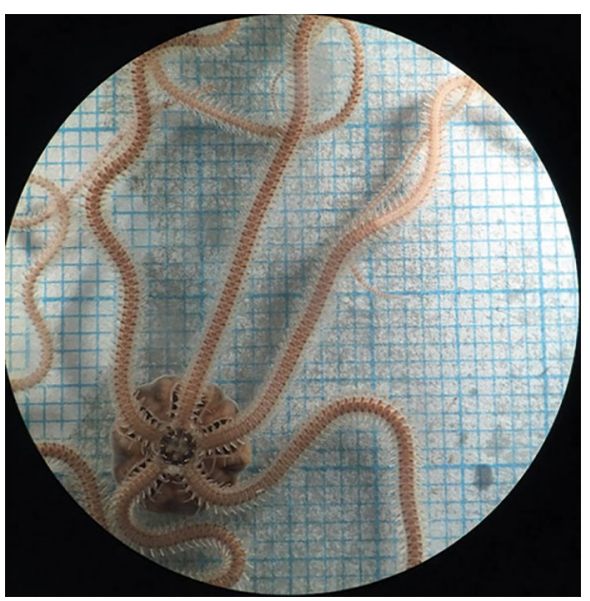

Oliveri lab, UCL. is a more typical animal genome, with large genes and big intergenic regions, both of which help with the study of complex regulation and other genome mechanisms ${ }^{3}$.

Intrigued by organismal diversity, she explores how the molecular underpinnings of major evolutionary transitions can be "read from the genome." As organisms evolve, their developmental networks, physiology and gene expression are rewired. In humans or primates, disentangling such rewiring is complicated. Echinoderms give access to embryos and organisms that develop quickly. With modern tools such as CRISPR for generating knockouts and knock-ins, she and her team explore how a regulatory network has changed in the course of evolution, says Oliveri.

Some labs focus on what is common across organisms, such as the homeobox genes that encode the anteroposterior body plan in many organisms. We all ultimately have the same ancestor, but evolution is not just what all have in common and has not changed, "it's also about novelty," she says. Echinoderms show that evolution generates novelty, she says, in that they have completely reshaped the body plan from a bilateral ancestor to life as a pentaradial organism, "which is absolutely dramatic."

\section{Mouse lemur, Microcebus murinus}

Models have been useful for understanding mechanism, such as sound localization in the owl. But humans do not have such a highly developed localization system. Mice have helped with understanding molecular mechanisms, but brain circuits in the mouse vision system are perhaps unlike the human one, he says. "Just because it's easy and it works, is it a good model system? I don't know. It's a system, but I don't think it's ideal," says Daniel Huber of the University of Geneva.

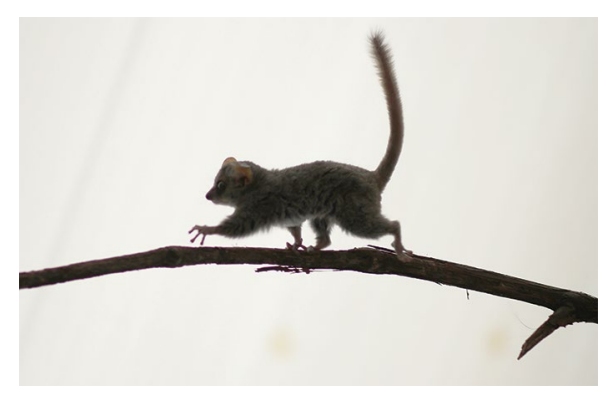

D. Huber, U. of Geneva.

Over the last four years he and his team have worked with the mouse lemur and found it is indeed an ideal species for neuroscience research, says Huber. The animal is small, easy to hold and handle, quick to train and pleasant to work with. Most cutting-edge neuroscience tools, such as optical imaging methods and molecular tools such as viral vectors that were initially developed for mice, could be readily adapted.

Using these tools, the team has shown ${ }^{4}$ that "despite their small size, their functional brain organization carries most hallmarks of the brains of larger primates," he says. The organization of the mouse lemur brain, as is the case with other primates, more closely resembles that of the human brain than the rodent brain in terms of circuits, structures and cell numbers per computational unit. In his psychophysics work on reactions to vibrational cues, in which he compares mice, mouse lemurs and people, the mouse results are usually quite unlike the mouse lemur and human results, he says. He meets scientists who "are intrigued and curious to hear more about the mouse lemur," says Huber. But they stick with the mouse, it seems, out of convenience rather than for a particular scientific reason. "So we end up with too many labs chasing exactly the same, often incremental, questions with the same methods in the same species," he says. Mouse lemurs show brain shrinkage as they age, they suffer cognitive decline, and their brains have tangles and plaques similar to those in the brains of people who had Alzheimer disease. When modeling such challenging conditions, he says, instead of inducing them in a model that does not suffer neurodegeneration, one could potentially benefit from studying naturally occurring conditions in mouse lemurs.

Huber and his team have also engineered a system ${ }^{5}$ to observe, perturb and condition behavior of freely roaming mouse lemurs. "Interacting with freely roaming animals in naturalistic environments has been captivating," he says. EthoLoop is an optical tracking system he set up amid a network of branches not unlike the tree branches mouse lemurs hop around in their native Madagascar. Beyond cameras, the system has reward boxes to encourage foraging behavior and mimic situations primate brains have actually evolved for, says Huber. Challenging and exploring the animals' capabilities can, he hopes, lead to a better understanding of how different brain areas function or interact during behavior. EthoLoop lets the team rapidly train mouse lemurs to carry out complex navigational or decision-making tasks as they climb around tree branches in complete darkness. The remote-controlled and remote-activated stimulus-reward boxes are stand-ins for flowers. Tiny peristaltic pumps pump out an occasional droplet of banana juice, which mirrors the plant nectar or fruit bits mouse lemurs forage for in the wild. In experiments, many labs reduce the degrees of freedom an animal might have 
in order to get reproducible results. With EthoLoop they can induce and capture repeated behavior or motor patterns in a reproducible way. "It's as if we bring the control into nature," he says.

\section{Filamentous fungus Ashbya gossypii}

One of Amy Gladfelter's lab videos shows a bushy structure with wavy, thin branches dotted with sparkling pearls. It's an under-the-microscope view of a single fungal cell with filaments that contain its many nuclei. The Gladfelter lab is at the University of North Carolina, Chapel Hill, and in the summers the team moves to the Marine Biological Laboratory in Woods Hole, Massachusetts. Ashbya gossypii is among the fungi Gladfelter studies. It's a plant pathogen that's closely related to the yeast Saccharomyces cerevisiae, a well-known model organism.

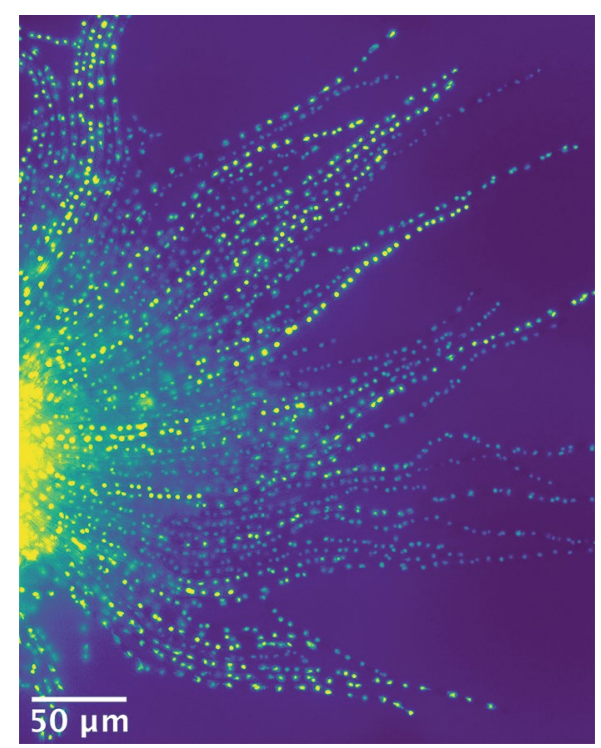

Gladfelter lab, T. Gerbich, G. McLaughlin/UNC Chapel Hill.

What fascinates Gladfelter about Ashbya is that its cells have many nuclei. This trait helps with exploring how the cytosol is spatially organized, which is one of her research questions. She also studies marine fungi, in terms of both their cell biology and their role in bigger contexts, such as how these fungi affect plankton and influence carbon cycling.

She and her team have developed a number of Ashbya protocols, such as ones for immunofluorescence staining, for single-molecule fluorescence in situ hybridization (FISH) and GFP boosting, for making media suited to Ashbya and for western blotting of Ashbya cultures. One aspect of interest is, she says, looking at the "whole milieu of small molecules" that engage with condensates, whether they are natural metabolites or drug candidates. Condensates are areas of the cytoplasm with liquid-liquid phase separation. It intrigues her to study how the nuclei in these fungal multinucleated cells behave. "That sort of sets up some really interesting cell biology questions, when you have multiple copies of the genome within a single cell." Some cells in our body are also multinucleated, such as muscle cells and those in the syncytiotrophoblast of the placenta. In her view, multinucleation may have evolved as a way to have "cells within cells," which can, for example, help a cell synchronize its activities.

With molecular genetic tools and CRISPR-based ones, researchers probe Ashbya for a number of questions in cell and developmental biology. Gladfelter is intrigued by phase separation, the way proteins congregate in the cytoplasm's condensates. "My favorite model condensate, the one we study in Ashbya," she says, can't be purified intact, which is why she does not yet have a catalog of its contents. Condensate research projects can start in silico or in a simple system and then move on to probe condensates in a live-cell environment. "I think this is a real frontier," she says, and one for which new methods are needed.

Condensates are replete with proteins and many RNA species. There is interaction between proteins and between proteins and RNAs, which makes condensates complex, and these interactions are part of how a condensate's molecular identity is specified, she says.

\section{Medaka, Oryzias latipes}

Although he also works with zebrafish, says Qiang Tu of the Institute of Genetics and Developmental Biology, which is part of the Chinese Academy of Sciences in Beijing, he mainly works with medaka, a much less well-known model organism. The medaka, or Oryzias latipes, is small, like zebrafish, and its embryos are transparent, as in zebrafish. The fish can be genetically manipulated, and mutagenesis is straightforward.

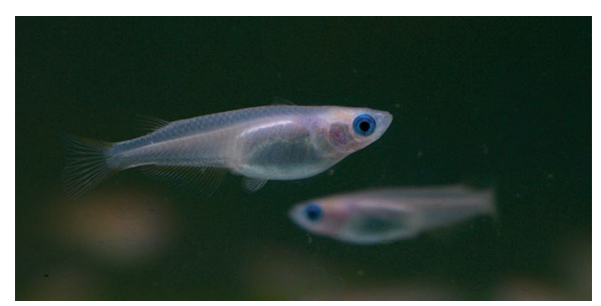

Tu lab, IGDB, CAS.

Tu was drawn to medaka as a model when he wanted to study gene regulatory networks during development. "I need a relatively simple and clearly defined biological system," he says, and he likes medaka's germ cell development. Zebrafish's complicated sex-determination system involves multiple, still unknown, genes and environmental factors, whereas medaka's genetic sex-determination system resembles the one in mammals. Some medaka strains express a pigment allele early in development that is tightly associated with the sex-determining gene, he says, "so you can distinguish the genetic gender by simply watching them under the microscope."

Medaka and zebrafish's last common ancestor lived more than 200 million years ago, which leads to important differences between the fishes. Medaka is fine with water temperatures down to $4{ }^{\circ} \mathrm{C}$, whereas zebrafish needs tropical temperatures above $20^{\circ} \mathrm{C}$. Medaka is quite tolerant to inbreeding and many inbred strains exist for lab use. The National BioResource Project funded by the Japan Agency for Medical Research and Development maintains over 600 strains, he says, including wild populations and inbred lines from wild populations. A near-isogenic inbred panel of 98 lines from the wild is in development. Such strains are powerful for studying gene-environment interaction. Tu has also worked with sea urchin, which suited his work on the gene regulatory network that underpins embryonic development. Using fluorescence microscopy of medaka, he can watch live germ cell development and know the genders. Zebrafish can regenerate heart tissue that is severely damaged, which makes zebrafish a good system for studying that process. None of these systems replace one another, he says.

Given the diversity of life on Earth, each system is a unique opportunity to observe and understand different aspects, says Tu. Labs should choose their research organism on the basis of their question of interest and keep in mind how essential diversity is for stable ecosystems. "We shouldn't narrow our scientific scope to a few 'model organisms,' he says. That's like, in line with a Chinese idiom, observing a leopard through a narrow tube.

The speedy advance of sequencing and 'omics technologies has eased the way for researchers to explore 'non-model organisms'. But a chasm exists between the resources available for major versus minor systems. The limited resources, he says, can be used well with careful strategizing. He suggests that labs look at the vast amount of human and mouse data generated to see what can be achieved with which techniques and their pros and cons. Next is to select critical technologies to apply to their systems of interest.

Medaka's compact genome is another positive: at 800 megabases, it's half the size of the zebrafish genome. "As I heavily depend on 'omics technologies, I thought 
a smaller genome might save me a lot of sequencing cost." But then he looked at the US National Institutes of Health's datasets of gene expression data and did a double-take. "Oops," he says: zebrafish has around 50 times more published 'omics datasets than medaka. The strategy he developed in response is one he calls "the minimum ENCODE toolbox ${ }^{6}$." This toolbox uses a combination of omics technologies - long-read RNA-seq, short-read RNA-seq, ATAC-seq — to initially profile organisms for which limited data are available. These methods are easy to use and yield rich information about different genomic aspects. "This combinatorial 'omics profiling was actually the first project I did when I turned to medaka, and all further projects greatly benefit from these datasets," he says.

\section{Clonal raider ant, Ooceraea biroi}

He's been called an ant paparazzo and doesn't mind the nickname, says Rockefeller University biologist Daniel Kronauer, who won a photography award from London's Natural History Museum for a photo shot in Costa Rica's northeastern rainforest. It shows a crown-shaped army ant nest, a bivouac that reaches across the stems of a leafy plant and teems with perhaps 100,000 army ants.

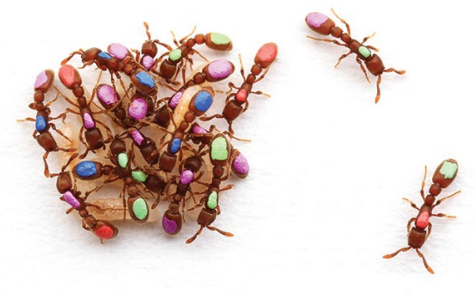

D. Kronauer.

Ants are Kronauer's model organism of choice $^{7}$. The species he and his team mainly work on is Ooceraea biroi, also known as the clonal raider ant ${ }^{8}$. Among insects, Drosophila melanogaster is certainly the model organism many labs choose, he says, but "there are many really interesting biological phenomena out there that can't be studied in fruit flies." One example is the highly sophisticated social behavior of eusocial insects, which include all ants and termites and some bees and wasps. What makes the clonal raider ant great for his work, he says, is that it shows some of the fascinating behavior of army ants, but lives in much smaller colonies that can be easily maintained in the lab. "By working with such unconventional model organisms, we can study questions that are not accessible in classical model systems," he says. Working with a new model system means developing a lot of new tools and protocols. In the case of this model organism, those have been mainly tools for behavioral analyses and protocols for genetic manipulations, he says.

On the subject of more basic biology, researchers now sometimes find that what was thought to apply to all insects on the basis of work from Drosophila is actually not generalizable. For example, he says, the development of the ant olfactory system differs in important ways from that of fruit flies.

Clonal raider ants are tiny, around 2.5 $\mathrm{mm}$ long, so they can be kept in colonies that fit in a Petri dish and the lab can conduct experiments with many replicates. He and his team built a setup that lets the scientists simultaneously film over a hundred colonies and track their behavior. Software recognizes each ant by a unique combination of color dots "that we painstakingly paint on the ants' back," says Kronauer. This system delivers a wealth of behavioral data. Among other findings, his group, along with colleagues at Princeton University, have found that that stable division of labor arises in quite small groups and increases with group size. "These kinds of studies can tell us something about the social dynamics during the early stages of group living, and why group living might be adaptive," he says. He also teams up with theoreticians to model the observed behavioral dynamics. It's a way to gain insights into which parameters are important in determining "who does what in an insect society."

In Kronauer's view, it's important for labs to broaden their scope in terms of study organisms. Historically, people had been working on much more diverse organisms, but as molecular genetics arose, most biologists believed it necessary to organize in communities around a handful of model species in which cutting-edge techniques in functional genetics and neuroscience could be developed, he says. More recent technological breakthroughs in DNA sequencing and editing make it possible to implement some of these techniques more broadly, he says. "So if you have a penchant for exotic creatures and want to do something different, this is a great time to be a scientist, " he says.

\section{Nematode Pristionchus pacificus}

It's a genetically tractable organism, which is an important reason why Sydney Brenner at the Medical Research Council's Laboratory of Molecular Biology studied nervous system development in Caenorhabditis elegans. John Sulston joined Brenner's group, and later he and colleagues performed manual lineage tracing of the entire organism. As Sulston recounts in his 2002 Nobel lecture, instead of holding the nematodes down, he let them crawl between the top of a thin, flat layer of agar and the cover slip and scraped a thin coating of bacteria - a nematode snack - onto the center of the cover slip. That snack coaxed the nematodes to stay in the area, "browsing gently along at a pace that allowed me to watch and draw the cells," Sulston said. "Seeing my first cell division was an exciting moment, because it implied that determining the larval lineage was possible. And indeed so it proved."

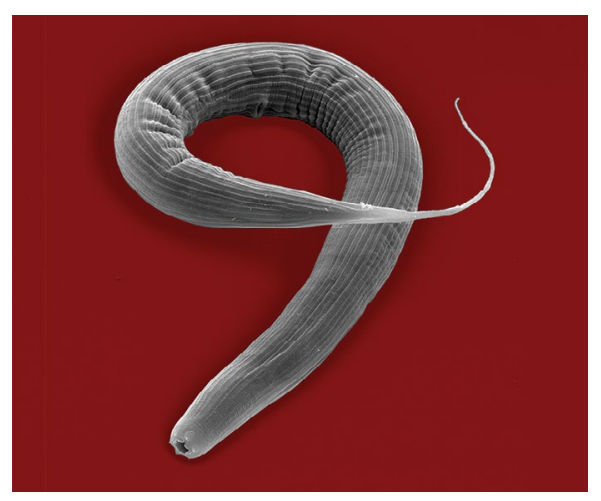

Sommer lab, MPIDB.

C. elegans is one of the major model organisms that thousands of labs around the world work on, says Ralf Sommer, a researcher at the Max Planck Institute for Developmental Biology. C. elegans meetings convene every other year. In 2013, he organized one at UCLA and had a full house of 1,800 participants. Sommer now spends more time on the nematode Pristionchus pacificus. He and his lab study genetics and epigenetics of this worm's predatory behavior.

Sommer established P. pacificus as a satellite organism of C. elegans. In the mid1990s, when evolutionary developmental biology, or 'evo-devo, was emerging as a field, he introduced the term 'satellite organism', which nowadays has "been a bit replaced by 'emerging' model systems," he says. "A long time ago," as he completed his $\mathrm{PhD}$ research in insects, he brought evo-devo to his nematode work. The idea behind evo-devo, he says, was to use the superb understanding of primary models, such as Drosophila and C. elegans, to the study of other insects and nematodes to learn about the evolution of developmental mechanisms and how this might fuel diversity. Around a decade ago, he nudged his lab away from classic evo-devo work to focus on developmental plasticity. He wonders, for example, how $P$. pacificus's genotype enables it to alter its phenotype as the environment changes and how plasticity might enable diverse phenotypes, physiology and behaviors.

He and his team study mouth form plasticity in these worms. When Pristionchus is grown on a plate of solid agar and salts in the presence of ample bacteria to munch on, the worms tend to be predatory. But liquid 
culture with the same bacterial snacks will induce non-predatory forms. Its plasticity is shaped by a developmental switch gene that has turned out to be a complex locus, more of a gene network. Using forward genetics techniques, the group teased out the genetic basis of the phenotypes; they then applied reverse genetics and perturbed these genes to assay their roles.

Plasticity as a concept has faced decades of skepticism and neglect, says Sommer. It wasn't until molecular mechanisms that underpin plasticity were identified that this crucial evolutionary concept made headway. As Sommer notes ${ }^{9}$, in 1989 entomologist and theoretical biologist Mary Jane West-Eberhard sketched out how neo-Darwinian thinking "sidelines organismal responses to the environment as being of little significance for evolution, although they are so tremendously widespread." Novelty starts with environmentally responsive, developmentally plastic traits, which need to be investigated with techniques including comparative genomics.

The Sommer lab's major finding took place when they compared vulva development in $C$. elegans and P. pacificus. Vulva development, he says, is one of the prime developmental processes and is extensively studied in C. elegans. The two nematodes have homologous vulvas. Despite the fact that the cells are the same, however, "the regulatory logic is fundamentally different," he says. In C. elegans, epidermal growth factor (EGF) signaling is the primary inductive force for vulva development, whereas in Pristionchus it's Wnt signaling. "This is maybe the best example of developmental systems drift," he says. What's seen in Pristionchus mouth-form plasticity, predation and self-recognition - are features specific to this model organism that $C$. elegans lacks. The scientists can use the same molecular tools on both, which, he says, helps the team identify molecular mechanisms.

\section{Redwoods, Sequoioideae}

Redwoods make up an ancient taxon: there's the coast redwood (Sequoia sempervirens) and the giant sequoia (Sequoiadendron giganteum), which both grow in California, and the dawn redwood (Metasequoia glyptostroboides), which grows only in China.

What's unique about long-lived forest trees is their ability to adapt to their environment, says David Neale, professor emeritus at the University of California, Davis. A research question important to him is how long-lived forest trees - as opposed to annual plants - manage to change and adapt. This is why these trees "can serve as models for adaptability and longevity, certainly in the plant kingdom," he says. Trees adapt to a

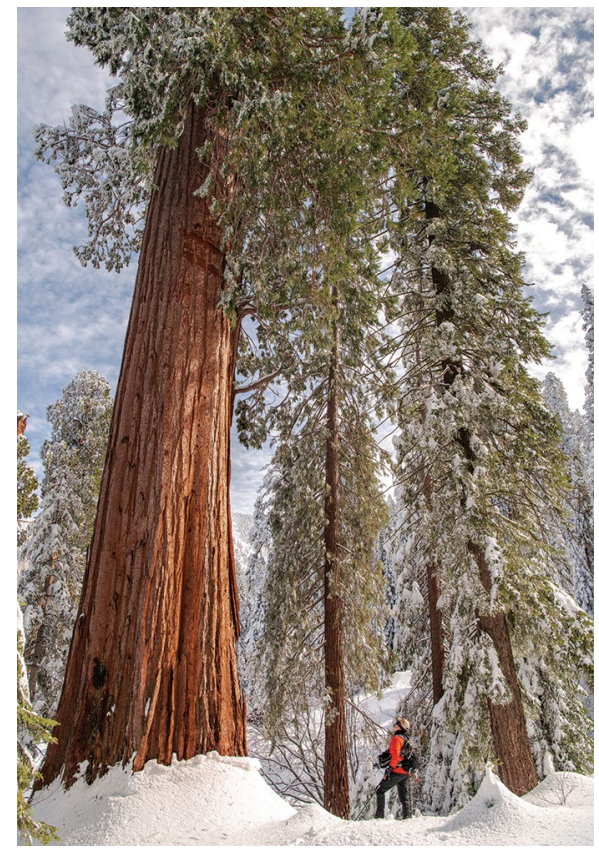

V. Reeder, Save the Redwoods League.

changing climate; after all, they "can't move and it takes a while for them to disperse naturally," he says. These trees offer much opportunity to gain basic knowledge related to adaptation and longevity.

In California, 95\% of old-growth redwood forests have been destroyed. This motivates some researchers to study "assisted migration," says Neale, which is thinking about how to repopulate forests. But without understanding the genetics and the needs of the trees, it is hard to know, for example, whether they can survive and thrive in other regions. Beyond latitudinal shifts, researchers want to study adaptation to drier environments. Given climate change, genomic data can help to understand which tree populations will have an easier and which a harder time.

The giant sequoia can grow to around 90 meters tall and can live to be more than 3,000 years old; the coast redwood can grow to over 110 meters in height and live to be around 2,000 years old. These mega-trees have mega-genomes, as Johns Hopkins University researcher Steven Salzberg phrased it in a talk on sequencing and assembling these genomes in front of users of Oxford Nanopore Technologies sequencers.

Salzberg and others at Johns Hopkins are part of the Redwood Genome Project, initiated by Neale and funded by the Save the Redwoods League. They sequenced the two trees' genomes and addressed their genomic complexity: the trees have large amounts of highly repetitive DNA, which made assembling these genomes quite challenging. The giant sequoia genome, published $^{10}$ in 2020, was completed with a combination of Illumina short read sequencing, Oxford Nanopore and Dovetail Genomics chromosome conformation capture technology. The coast redwood genome is in the works, says Neale. There is much to reveal about redwoods, he says. One approach might be to use comparative genetics to look at long-lived and short-lived trees side by side. It matters to understand the redwoods and their highly complex genomes better. This can help us conserve and restore redwood forests and understand how to maintain genetic diversity of the trees in the face of logging and climate change. The coast redwood genome is hexaploid and it intrigues him to understand when in the trees' evolution this multiplication of chromosomes happened. There might have been copying events or perhaps hybridization, as is ubiquitous in angiosperms - a plant group full of polyploids. "On the gymnosperm side, it's quite rare," he says, and among conifers, polyploidy makes the redwoods unique.

Neale has spent his career on the genetics of forest trees, which have not been considered models because they're hard to work with, he says. They're long-lived, it's not easy to make crosses and their genomes are large. This is the opposite of the attributes of the classic model plant Arabidopsis thaliana, which has a small genome; the plant can be easily clonally propagated and crosses are quickly done in two weeks in a greenhouse. Earlier sequencing technology kept labs away from tree mega-genomes. "It was just off the table because of the cost," says Neale.

At a conference, he recalls disagreeing with an Arabidopsis geneticist who held the view that everything about adaptation could be learned from Arabidopsis. "I don't really think so," Neale remembers saying. These days that community is much more receptive, he says. "Technology has leveled the playing field."
Vivien Marx ${ }^{凶}$
Nature Methods.

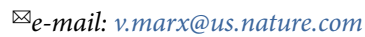

Published online: 24 February 2021 https://doi.org/10.1038/s41592-021-01086-7

References

1. Davis, R. H. Nat. Rev. Genet. 5, 69-76 (2004)

2. Sánchez Alvarado, A. Dev. Biol. 433, 111-114 (2018).

3. Arnone, O., Oliveri, P. \& Martinez, P. Mar. Genomics 44, 24-31 (2019).

4. Ho, C. L. A. et al. Curr. Biol. 31, 1-9 (2021)

5. Nourizonoz, A. et al. Nat. Methods 17, 1052-1059 (2020).

6. Li, Y. et al. Genome Res. 30, $924-937$ (2020).

7. Ulrich, Y., Saragosti, J., Tokita, C. K., Tarnita, C. E. \& Kronauer, D. J. C. Nature 560, 635-638 (2018).

8. Kronauer, D. Army Ants: Nature's Ultimate Social Hunters (Harvard Univ. Press, 2020).

9. Sommer, R. Genetics https://doi.org/10.1534/genetics.120.303163 (2020).

10. Scott, A. D. et al. G3 (Bethesda) 10, 3907-3919 (2020). 